\title{
The development and theoretical application of an implementation framework for dialectical behaviour therapy: a critical literature review
}

\author{
Gill Toms ${ }^{1}$, Lynne Williams ${ }^{1}$, Jo Rycroft-Malone ${ }^{1}$, Michaela Swales ${ }^{2 *}$ and Janet Feigenbaum ${ }^{3}$
}

\begin{abstract}
Background: Dialectical behaviour therapy (DBT) is a third wave behaviour therapy combining behaviour based components with elements of mindfulness. Although DBT effectiveness has been explored, relatively little attention has been given to its implementation. Frameworks are often the basis for gathering information about implementation and can also direct how the implementation of an intervention is conducted. Using existing implementation frameworks, this critical literature review scoped the DBT implementation literature to develop and refine a bespoke DBT implementation framework.

Method and results: The initial framework was developed by consolidating existing implementation frameworks and published guidance on DBT implementation. The critical literature review retrieved papers from Medline, CINAHL, Psyclnfo, PubMed, and the reference lists of included papers. Framework elements were used as codes which were applied to the literature and guided the synthesis. Findings from the synthesis refined the framework. The critical literature review retrieved 60 papers but only 14 of these explicitly focused on implementation. The DBT implementation framework captured all the execution barriers and facilitators described in the literature. However, the evidence synthesis led to a more parsimonious framework as some elements (e.g., research and published guidance) were seldom discussed in DBT implementation.

Conclusion: To our knowledge this is the first published review exploring DBT implementation. The literature synthesis suggests some tentative recommendations which warrant further exploration. For instance, if DBT implementation is not pre-planned, having someone in the organisation who champions DBT can be advantageous. However, as the literature is limited and has methodological limitations, further prospective studies of DBT implementation are needed.
\end{abstract}

Keywords: Dialectical behaviour therapy, Implementation, Psychological therapy, Review

\section{Background}

Dialectical Behaviour Therapy (DBT) [1] synthesises behavioural based therapy components (orientated towards change) with elements from mindfulness (orientated towards increasing acceptance). DBT is typically offered to people with a diagnosis of Borderline Personality Disorder (BPD) and a history of suicidal and self-harming

\footnotetext{
* Correspondence: m.swales@bangor.ac.uk

${ }^{2}$ North Wales Clinical Psychology Programme, School of Psychology,

Brigantia Building, Bangor University, Bangor, Gwynedd LL57 2DG, UK

Full list of author information is available at the end of the article
}

behaviour. Therapists aim to impart new skills and develop clients' behavioural flexibility to draw on appropriate skills in any given social or emotional situation. Core treatment components include individual therapy, telephone skills coaching, skills group and a clinician consultation team (where DBT therapists access support and guidance from other DBT team members), although services may only deliver some of these components (e.g. [2]). Several reviews summarising the evidence for DBT effectiveness are available (e.g. [3, 4]).

(c) The Author(s). 2019 Open Access This article is distributed under the terms of the Creative Commons Attribution 4.0 International License (http://creativecommons.org/licenses/by/4.0/), which permits unrestricted use, distribution, and 
Implementation is the process through which the uptake of evidence-based interventions in routine clinical practice is systematically promoted. Transdisciplinary implementation frameworks exist, for instance, Promoting Action on Research Implementation in Health Services (PARIHS: [5, 6]), the Consolidated Framework for implementation for implementation research (CFIR: [7]), and the Core Implementation Components model $([8,9])$. The PARIHS framework covers many of the core elements of these models: context, evidence, facilitation and intervention elements. Context refers to the environment or setting that the implementation takes place in. Evidence can be derived from research, clinical experience or patient preference. Facilitation refers to the people and processes that support implementation and the intervention element demotes the characteristics of the intervention to be implemented. CFIR has an additional element related to implementation processes, which describes the practical implementation tasks undertaken. Each of these elements are sub-divided. For instance, PARIHS sub-divides evidence into research and published guidance, clinical experience and professional knowledge, preferences and experiences, and local knowledge. National implementations refer to many of these elements in their guidance, for example, the Increasing Access to Psychological Therapies manual [10].

DBT has unique features, such as, a multicomponent therapy process, telephone skills coaching, and a consultation team. The characteristics of people with BPD (the core client group) could also necessitate bespoke implementation strategies. The question of how best to implement a DBT intervention arose in the context of the Enabling and Motivating people (with a Personality Disorder) in Occupation, Wellbeing, Education and Responsibility (EMPOWER) research programme (NIHR Programme Grant: RP-PG-1212-20,011), which is developing and evaluating a DBT- Skills for Employment (DBT-SE) intervention. The evidence about DBT implementation has seldom been reviewed and this work was undertaken with a view to developing an implementation toolkit for the DBT-SE intervention. We aimed to review the DBT implementation literature to develop and refine a bespoke DBT implementation framework.

\section{Methods}

\section{Framework development}

To create an initial DBT implementation framework, elements from the main transdisciplinary frameworks (PARIHS, CFIR, and the Core Implementation Components model) and from published DBT implementation guidance $[11,12]$ were synthesised. PARIHS $[5,6]$ was selected as the underpinning framework as it highlights the pivotal role of contextual factors. Although it developed from existing models, this is the first framework to incorporate implementation insights from the DBT literature. The initial framework is represented in Fig. 1 and is described in Additional file 1.

\section{Critical literature review}

To refine the framework, a critical literature review [13] was conducted. In critical reviews, the synthesis process is used to create a new model or a model embodying existing theory which then provides a 'launch pad' for subsequent testing. One of the strengths of this type of review lies in the analysis undertaken to create the model [13].

Four databases were searched with the terms 'DBT' and 'Implementation' in January 2016; Medline (EBSCO), CINAHL (EBSCO), PsycInfo (ProQuest), and PubMed (NCBI). These databases were selected as they hold health and psychology related literature. Reference lists of included papers were additionally screened as implementation issues might be discussed without this term being used as a key word or included in the abstract or title. DBT was defined as any combination of components or interventions which were identified as DBT by the study authors (Additional file 2 contains an example search). Implementation was defined as the process of introducing and sustaining DBT in routine practice. All retrievals were managed in RefWorks, an online bibliographic management programme. Only peer-reviewed papers were included but no date or evidence type restrictions were applied. For resource and time reasons, only papers published in English were included. The first author conducted the review and the eligibility of database retrieved papers was checked by a second reviewer (reviewer agreement was 97\% with all disagreements resolved through discussion).

Consistent with the critical review approach, papers were not excluded for methodological reasons [13]. However, prospective and retrospective studies of implementation were considered to provide the strongest evidence due to their explicit focus on implementation. Discussion pieces were judged to form the weakest evidence as the experiences they are based on are often not accessible for review. The data extracted from papers included; the design, context, methodology, implementation barriers and facilitators, as well as author conclusions and recommendations (Additional file 3 contains the data extraction template). Extracted data were discussed by the review team and where necessary the text was re-reviewed.

\section{Evidence synthesis}

Extracted data relating to implementation barriers and facilitators, conclusions and recommendations were coded using deductive content analysis. This process used the elements and sub-elements in the DBT 


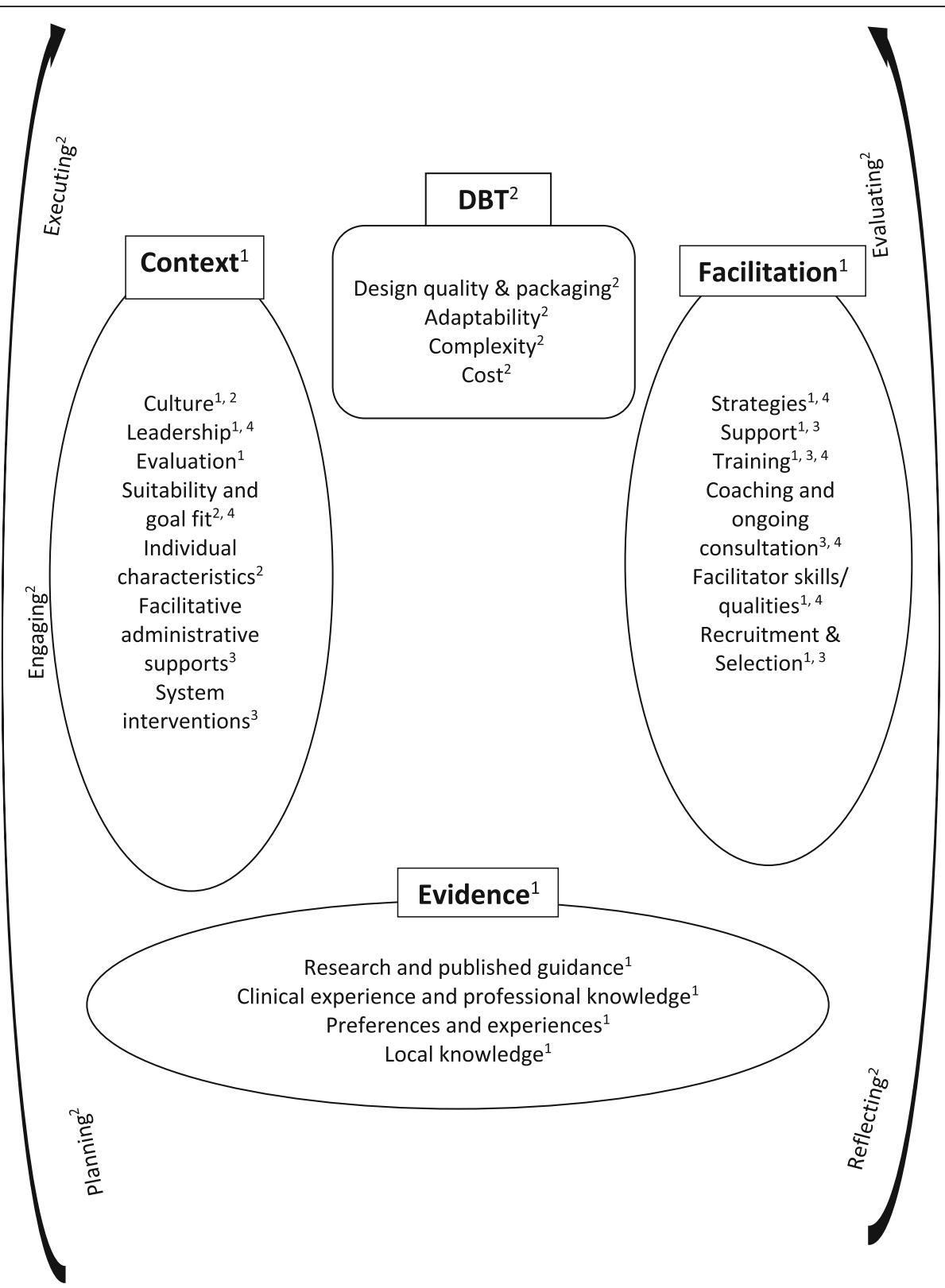

Fig. 1 DBT implementation framework: first iteration. Key: PARIHS, 2004; Damschroder et al., 2009²; Fixsen \& Blasé, 2009³ Swales 2010a, 2010b

implementation framework as code labels and assigned them to the data segments. Where the extracted data did not fit any existing codes, a new code name was added and this process continued until all the data were categorised. Coding was conducted by the first author and a second reviewer checked the coding applied to sixteen papers (10\% of the papers coded): although conservatively judged agreement was $66 \%$ (a criterion that the same sub-elements were coded in each paper), differences in coding were negligible and easily resolved through discussion. For instance, the most common cause for disagreement was which code best captured the data. The team reviewed the final synthesis to ensure it presented an accurate reflection of the data.

\section{Results}

\section{Critical literature review}

Sixty-two papers met the inclusion criteria (32 from database and 30 from reference list searches), although two papers were unobtainable within the time limit of the review. The main reasons for exclusion were failure to consider DBT or a failure to discuss implementation issues (see Fig. 2). There were 11 discussion papers ([11, 12, 14-22]) and as these were considered the weakest form of evidence 


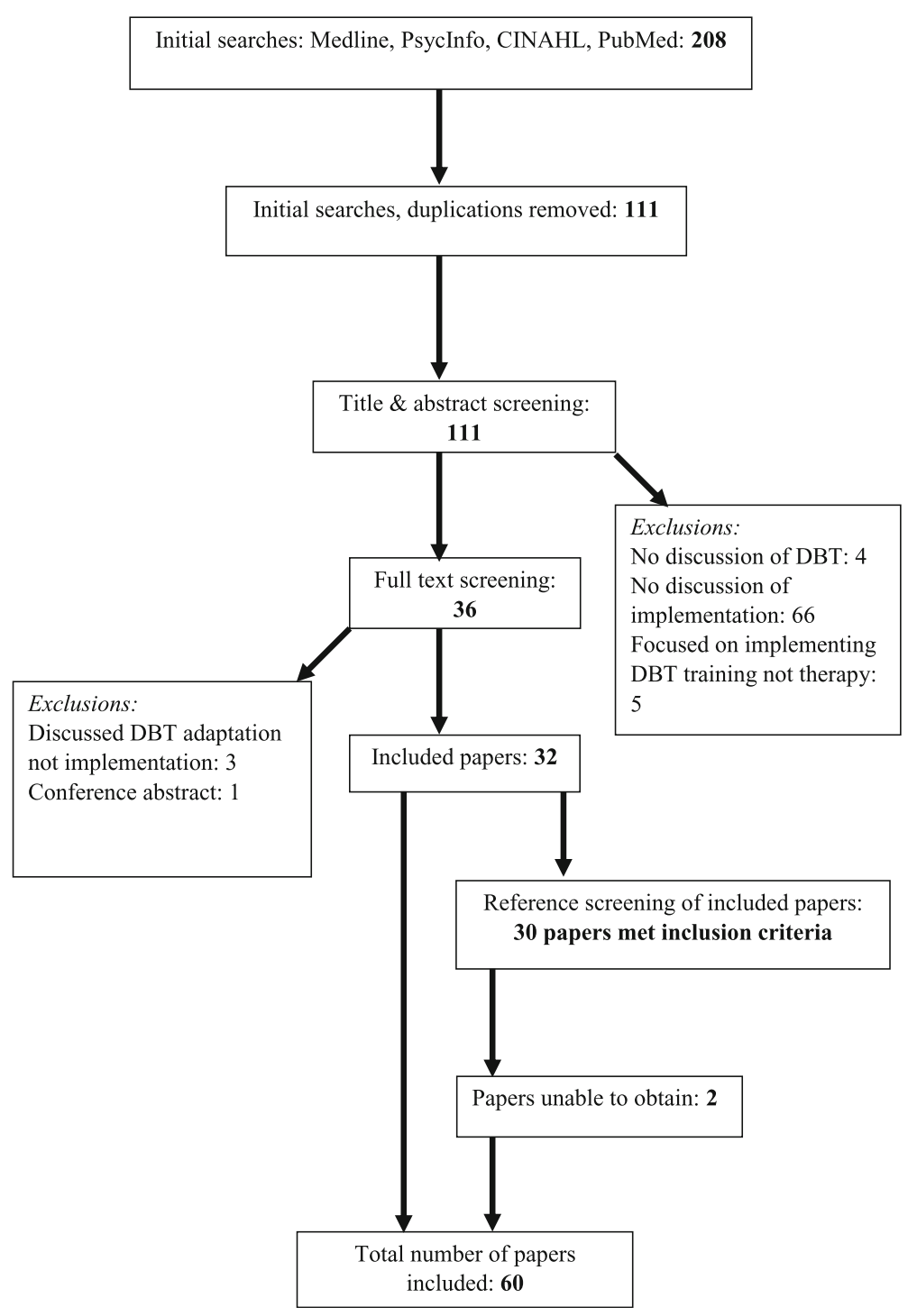

Fig. 2 Literature review flow chart

they were not included in the synthesis, but are detailed in Table 1. As seen in Table 2, nine papers collected retrospective ([23-31]) and five papers ([32-36]) collected prospective implementation data. There were 16 programme descriptions ([37-52]) and 19 trial process analyses $([53-71])$. The majority $(N=38)$ of papers were from the United States (US), and most implementations of DBT were in mental health services.

\section{Evidence synthesis}

Overall, 788 framework codes were assigned to the extracted data: 170 codes were allocated to studies specifically considering implementation, 209 codes to process analysis studies, 224 codes to programme descriptions, and the remainder were assigned to discussion papers (see Additional file 4). The DBT implementation framework is used to present the literature synthesis and, when possible, the data discussed is derived from the papers which explicitly studied implementation.

\section{Context}

Our initial DBT framework included seven context sub-elements (culture, leadership, evaluation, goal fit and suitability, individual characteristics, facilitative administrative supports and system interventions). Our synthesis of the literature yielded five primary sub-elements (culture, leadership, goal fit and suitability, facilitative administrative supports and system interventions), which are discussed below: 
Table 1 Discussion papers

\begin{tabular}{ll}
\hline Reference & Country \& service context \\
\hline Chugani, 2015 [16] & $\begin{array}{l}\text { America. College counselling } \\
\text { centres }\end{array}$ \\
& \\
$\begin{array}{ll}\text { Borroughs \& Somerville, } & \text { America. Assertive Community } \\
2013 \text { [15] } & \text { Treatment teams }\end{array}$
\end{tabular}
2013 [15]

Koener, 2013 [18]

McHugh \& Barlow 2010 [19]

Swales, 2010a [11]

Swales, 2010b [12]

Berzins \& Trestman, 2004 [14]

Huffman et al., 2003 [17]

Swenson et al., 2002 [21]
Treatment teams

N/A

Worldwide; Reviews and describes a range of implementation efforts

UK

UK

America. Prison/correctional services. Non-systematic review and information collected from services

N/A

America. Public mental health authorities. Recommendations based on observations, a survey and literature review
Key points/ recommendations made

-Important to collect service-relevant outcome data as DBT is often

adapted to fit the service

-Important to adapt DBT so appropriate for the service

-Can mitigate costs by hosting training or offering partial

programmes

-There may be resource and financial barriers, especially in the US

healthcare system where services cannot recoup costs for training,

consultation team meetings or data collection

-It is important to determine if DBT 'fits' the service's client group and theoretical stance

-Recommended adapting DBT and offsetting costs by using existing infrastructure and demonstrating cost-effectiveness

-DBT clinicians need a good conceptualisation of the therapy, including the treatment hierarchy and biosocial theory

-Important that therapists are dialectical, cognitively flexible and validating

-Recursive culture important; a community of therapists working with a community of patients, with everyone in the same boat

-Services need to see patients as motivated to change and that services want to improve patient capability

-Therapists should access the consultation team and mindfulness practice

-Ensuring fidelity to manualised DBT ignores the contextual factors that moderate success

-In America, Behavior Tech acts as a champion for DBT

--Ongoing outcome monitoring important to sustain fidelity and quality improvement -Implementation issues have informed DBT training. For instance, teams implement DBT before completing final training so that they can access consultation after their first attempts

-Larger DBT teams with less time will be slower at learning DBT than smaller teams who have greater allocated time

-Important to gain staff commitment to implement DBT and to select staff with knowledge about DBT and implementation, who are willing to apply DBT skills themselves

-Beneficial to recruit so that DBT teams encompass a range of skills

-Important to have a DBT 'champion' and the team leader should be in a senior position -Consultation teams have an important role and the consultation agreement establishes the team climate

-A minimum of two hours per work is necessary for supervision and consultation team meetings

-Description of an organisational pre-treatment approach where the DBT team leader or champion:

-Identifies the appropriateness of DBT, weighing the evidence, policy aims and organisation suitability, culture and climate

-Considers the organisations experience in implementing other new therapies -Resolves competing goals and if synthesis is impossible undertakes a pros and cons analysis

-Forms an advisory or steering group to address factors likely to interfere with implementation

-All the programmes described had adapted DBT. There is currently no manual for DBT in correctional settings

-Programmes were driven by clinical need (DBT had 'goal fit')

-To implement and evaluate a proposed modified DBT programme for correctional settings, a coalition had been formed between the university, state and health departments

-Champion/consultant should be willing to model DBT skills

-To accommodate time limitations, single components of DBT can be applied rather than the comprehensive intervention

-Need to provide psychoeducation about BPD and validate staff experience of difficulties

-Use contingency management; frame behaviour modification as the most effective approach

-Barriers listed included therapist view of DBT suitability and staff turnover. Discussed therapist selection issues

-Also discussed the barriers patients may face when starting DBT- e.g. it is a high time commitment and they might need to terminate current treatment contracts

-Facilitators endorsed leadership from public mental health authorities, training, a positive attitude towards BPD and monitoring outcomes

-Recommended forming coalitions between organisations providing DBT and those planning to implement DBT

-Recommended providing training (psychoeducation) for public mental health authorities about DBT and BPD

-Recommended highlighting to patients that DBT participation is voluntary 
Table 1 Discussion papers (Continued)

\begin{tabular}{lll}
\hline Reference & Country \& service context & Key points/ recommendations made \\
\hline Scheel, 2000 [20] & N/A. Overview and & -Suggested inpatient settings might transition most easily to DBT, as there is fit in \\
& literature critique & terms of time availability and goals \\
& -Need access to training, supervision and consultation \\
& -Implementing DBT in a manner consistent with the evidence base requires a \\
& considerable staff team: resources may threaten viability \\
& -Outpatient DBT requires inter-agency support (a need for coalitions) \\
Swenson, 2000 [22] & -Should use DBT skills to help implementation \\
& -The design of DBT contributes to its appeal to therapists. For instance, it integrates \\
& different orientations meaning it has a wide support base and therapists from various \\
& orientations automatically have 'buy-in' \\
& -DBT can be both pragmatic and very sophisticated \\
\hline
\end{tabular}

\section{Culture}

There are two elements of culture that capture staff behaviour within the organisation $[5,6]$ :

\section{Communication processes}

Better ratings of organisation cohesion and communication correlated with the implementation of more DBT components [26], perhaps because institutional adoption of DBT depends on the collaboration of many staff [34]. On-going external consultation helps achieve sustainable programmes [23] and good communication was important within the DBT consultation team [29]. There were examples of communication forming both a facilitator (e.g. [40]) and a barrier [46]. Communication within and across teams seemed particularly important when client characteristics, such as intellectual disability or offender status, meant collaborative working was essential (e.g. [45]).

\section{Climate}

Higher scores on the Team Climate Inventory correlated with the implementation of more DBT components [26] and limited understanding of staff and patients' needs could form a barrier [29]. The importance of team support was endorsed by therapists [28]. Attitudes toward BPD seemed key. A non-judgemental, validating stance seems necessary to create the right environment [25], and better attitudes towards BPD correlated with increased use of DBT [35]. In one survey negative administrator attitudes reportedly impeded implementation [23].

\section{Leadership}

In a therapist survey, one of the most common reasons for DBT team cessation was a lack of leadership or organisation 'buy-in'. Where team leadership was supportive, $19.6 \%$ of respondents said this facilitated implementation [31]. Respondents in another study similarly reported that a lack of understanding amongst service leaders constituted an implementation barrier [28]. Often the implementation of DBT had not been pre-planned and in these scenarios having a 'DBT champion' in the organisation seemed important. Champions needed to have influencing skills (e.g. [51]), cultural sensitivity, a willingness to undertake tasks, such as, securing funding [43], and an ability to model DBT skills [46]. In many cases the DBT consultation team seemed to undertake championing tasks through generating interest (e.g. [59]), establishing collaborations (e.g. [65]), offering expertise to other agencies (e.g. [52]) and providing support to the wider staff team (e.g. [69]).

\section{Goal fit and suitability}

Sometimes DBT was viewed positively from the outset [36] and greater confidence in DBT effectiveness correlated with increased use of DBT [35]. However, DBT was not always seen as suitable [24, 30]. DBT implementation was also weakened by competing service priorities [31]. For instance, in a substance abuse service, DBT was incompatible with the delivery model of short visits primarily providing methadone [23]. Some administrators were concerned about the telephone coaching component of DBT, as telephone support had not worked previously [36] and services need a minimum number of patients to run DBT groups [30]. However, whilst belief in DBT suitability and fit could facilitate implementation (e.g. [58]), the lack of this belief was not necessarily a barrier, as perceptions could change during the implementation process (e.g. [55]).

\section{Facilitative administrative supports}

Insufficient time could be a barrier, whereas the allocation of sufficient time could be a facilitator [31]: in one survey, $42 \%$ of therapists reported having a lack of time to provide DBT [23]. Some therapists talked about needing to divide their time between different tasks [28] and administrators were concerned that DBT training would keep staff from their clinical duties [36]. Other required resources were finances [36] and space: having adequate space correlated with the implementation of more DBT components [26]. The data also suggests that contingency management has the potential to influence implementation. For instance, organisations often failed to reduce other staff-held responsibilities to compensate for new DBT commitments [23] thereby punishing engagement in DBT. Enabling natural contingencies, such 
Table 2 Implementation papers, programme descriptions and trial process analysis papers

\begin{tabular}{|c|c|c|c|c|c|}
\hline Reference & Country \& service context & Paper type & Methodology & DBT outcomes & $\begin{array}{l}\text { Implementation } \\
\text { relevant outcomes }\end{array}$ \\
\hline \multicolumn{6}{|c|}{ Implementation papers } \\
\hline $\begin{array}{l}\text { Chwalek \& McKinney, } \\
2015 \text { [24] }\end{array}$ & $\begin{array}{l}\text { America (and Germany). } \\
\text { Range of mental health } \\
\text { services }\end{array}$ & $\begin{array}{l}\text { Retrospective } \\
\text { data collection }\end{array}$ & $\begin{array}{l}\text { Survey and interviews } \\
\text { of music therapists }\end{array}$ & N/A & $\begin{array}{l}\text { 38.3\% of respondents } \\
\text { endorsed implementing } \\
\text { DBT in music therapy } \\
\text { practice }\end{array}$ \\
\hline Ditty et al., 2015 [26] & $\begin{array}{l}\text { America. Mental } \\
\text { health services }\end{array}$ & $\begin{array}{l}\text { Retrospective } \\
\text { data collection }\end{array}$ & $\begin{array}{l}\text { Survey and interviews } \\
\text { with therapists trained } \\
\text { in DBT exploring inner } \\
\text { setting constructs of } \\
\text { CFIR framework }\end{array}$ & N/A & $\begin{array}{l}96 \% \text { of respondents } \\
\text { provided individual } \\
\text { therapy, } 99 \% \text { provided } \\
\text { skills groups, } 97 \% \text { attended } \\
\text { a consultation team and } \\
87 \% \text { provided phone skills } \\
\text { coaching }\end{array}$ \\
\hline $\begin{array}{l}\text { Carmel et al., } \\
2014 \text { [23] }\end{array}$ & $\begin{array}{l}\text { America. Public } \\
\text { behaviour } \\
\text { health system }\end{array}$ & $\begin{array}{l}\text { Retrospective } \\
\text { data collection }\end{array}$ & $\begin{array}{l}\text { Telephone interviews } \\
\text { with therapists }\end{array}$ & N/A & $\begin{array}{l}\text { Therapists received ten } \\
\text { days }(80 \mathrm{~h}) \text { of DBT training } \\
\text { over } 13 \text { months }\end{array}$ \\
\hline $\begin{array}{l}\text { Herschell et al., } \\
2014 \text { [35] }\end{array}$ & America & $\begin{array}{l}\text { Prospective } \\
\text { data collection }\end{array}$ & $\begin{array}{l}\text { Quantitative survey of } \\
\text { therapists pre and post } \\
\text { implementation }\end{array}$ & $\begin{array}{l}\text { Therapists reported trend } \\
\text { reduction in patient A\&E } \\
\text { visits and hospitalisations }\end{array}$ & $\begin{array}{l}\text { Therapist training ranged } \\
\text { from } 32 \text { to } 96 \mathrm{~h} \text { (maximum } \\
96 \mathrm{~h} \text { ) and received on } \\
\text { average } 25.67 \mathrm{~h} \text { of phone } \\
\text { consultation }\end{array}$ \\
\hline $\begin{array}{l}\text { Swales et al., } \\
2012 \text { [31] }\end{array}$ & $\begin{array}{l}\text { UK. Range of inpatient, } \\
\text { outpatient and forensic } \\
\text { services }\end{array}$ & $\begin{array}{l}\text { Retrospective } \\
\text { data collection }\end{array}$ & $\begin{array}{l}\text { Telephone interviews } \\
\text { with DBT team } \\
\text { members }\end{array}$ & $\begin{array}{l}7.1 \% \text { said improved } \\
\text { patient outcomes were an } \\
\text { implementation facilitator }\end{array}$ & $\begin{array}{l}62.8 \% \text { of programmes } \\
\text { remained active at five } \\
\text { years. } 57 \% \text { of programmes } \\
\text { provided all DBT } \\
\text { components }\end{array}$ \\
\hline $\begin{array}{l}\text { Dimeff et al., } \\
2011[32]\end{array}$ & America. & $\begin{array}{l}\text { Prospective } \\
\text { data collection }\end{array}$ & $\begin{array}{l}\text { Randomised controlled } \\
\text { trial with DBT naive } \\
\text { therapists }\end{array}$ & N/A & $\begin{array}{l}\text { E-learning resulted in best } \\
\text { knowledge retention at } 15 \\
\text { week follow-up }\end{array}$ \\
\hline $\begin{array}{l}\text { Dimeff et al., } \\
2009[33]\end{array}$ & America. & $\begin{array}{l}\text { Prospective } \\
\text { data collection }\end{array}$ & $\begin{array}{l}\text { Randomised controlled } \\
\text { trial with DBT naïve } \\
\text { therapists }\end{array}$ & N/A & $\begin{array}{l}80 \% \text { of therapists } \\
\text { completed training. Online } \\
\text { training best at improving } \\
\text { knowledge. Instructor led } \\
\text { training better than } \\
\text { reading the training } \\
\text { manual at increasing self- } \\
\text { efficacy and satisfaction }\end{array}$ \\
\hline $\begin{array}{l}\text { Herschell et al., } \\
2009 \text { [36] }\end{array}$ & $\begin{array}{l}\text { America. Community } \\
\text { mental health services }\end{array}$ & $\begin{array}{l}\text { Prospective } \\
\text { data collection }\end{array}$ & $\begin{array}{l}\text { Qualitative interviews pre } \\
\text { implementation with } \\
\text { county level mental health } \\
\text { administrators }\end{array}$ & N/A & $\mathrm{N} / \mathrm{A}$ \\
\hline $\begin{array}{l}\text { Perseuis et al., } \\
2007 \text { [28] }\end{array}$ & $\begin{array}{l}\text { Sweden. Outpatient } \\
\text { services }\end{array}$ & $\begin{array}{l}\text { Retrospective } \\
\text { data collection }\end{array}$ & $\begin{array}{l}\text { Survey and interviews } \\
\text { with DBT trained } \\
\text { therapists }\end{array}$ & N/A & $\begin{array}{l}\text { Therapists worked } \\
\text { part-time in the DBT team. } \\
\text { Tendency for greater staff } \\
\text { burnout over time, but not } \\
\text { statistically significant. } \\
\text { Reduced } \\
\text { occupational stress }\end{array}$ \\
\hline $\begin{array}{l}\text { Sharma et al., } \\
2007 \text { [30] }\end{array}$ & $\begin{array}{l}\text { America. Psychiatric } \\
\text { residency }\end{array}$ & $\begin{array}{l}\text { Retrospective } \\
\text { data collection }\end{array}$ & $\begin{array}{l}\text { Survey of residency } \\
\text { directors and senior } \\
\text { residents. Also } \\
\text { presented a } \\
\text { case study }\end{array}$ & $\begin{array}{l}\text { Patient hospitalised then } \\
\text { discontinued DBT therapy }\end{array}$ & $\begin{array}{l}56 \% \text { of residency } \\
\text { programmes had no } \\
\text { lectures on DBT and 32\% } \\
\text { provided no DBT } \\
\text { supervision }\end{array}$ \\
\hline $\begin{array}{l}\text { Frederick \& } \\
\text { Comtois, } 2006 \text { [27] }\end{array}$ & America & $\begin{array}{l}\text { Retrospective } \\
\text { data collection }\end{array}$ & $\begin{array}{l}\text { Survey of psychiatry } \\
\text { residency graduates } \\
\text { who had attended at } \\
\text { least one DBT workshop }\end{array}$ & N/A & $\begin{array}{l}23 \% \text { of respondents } \\
\text { practiced all DBT } \\
\text { components. Most } \\
\text { practiced at least one } \\
\text { DBT component }\end{array}$ \\
\hline $\begin{array}{l}\text { Cunningham et al., } \\
2004 \text { [25] }\end{array}$ & America & $\begin{array}{l}\text { Retrospective } \\
\text { data collection }\end{array}$ & $\begin{array}{l}\text { Interviews with BPD } \\
\text { patients who had } \\
\text { received DBT therapy }\end{array}$ & $\begin{array}{l}\text { Reduced hospitalisations } \\
\text { and increased vocational } \\
\text { work }\end{array}$ & N/A \\
\hline
\end{tabular}


Table 2 Implementation papers, programme descriptions and trial process analysis papers (Continued)

\begin{tabular}{|c|c|c|c|c|c|}
\hline Reference & Country \& service context & Paper type & Methodology & DBT outcomes & $\begin{array}{l}\text { Implementation } \\
\text { relevant outcomes }\end{array}$ \\
\hline $\begin{array}{l}\text { Perseius et al., } \\
2003 \text { [29] }\end{array}$ & Sweden & $\begin{array}{l}\text { Retrospective } \\
\text { data collection }\end{array}$ & $\begin{array}{l}\text { Interviews with DBT } \\
\text { therapists and patients }\end{array}$ & $\begin{array}{l}\text { Patients reported positive } \\
\text { outcomes. Patients had } \\
\text { been in therapy for at } \\
\text { least } 12 \text { months }\end{array}$ & $\begin{array}{l}\text { Therapists gained a new } \\
\text { perspective and DBT } \\
\text { influenced how therapists } \\
\text { solved problems in their } \\
\text { own lives }\end{array}$ \\
\hline \multirow[t]{2}{*}{$\begin{array}{l}\text { Hawkins \& Sinha, } \\
1998[34]\end{array}$} & $\begin{array}{l}\text { America. Department } \\
\text { of mental health and } \\
\text { addiction services }\end{array}$ & $\begin{array}{l}\text { Prospective } \\
\text { data collection }\end{array}$ & $\begin{array}{l}\text { Correlated therapist } \\
\text { DBT knowledge to } \\
\text { demographics and } \\
\text { training through } \\
\text { repeated measures } \\
\text { and naturalistic service } \\
\text { outcome data }\end{array}$ & $\begin{array}{l}\text { Archival data suggested } \\
\text { DBT training led to better } \\
\text { patient outcomes: less } \\
\text { A\&E, inpatient, seclusion } \\
\text { and restraint use }\end{array}$ & $\begin{array}{l}\text { Training and the amount } \\
\text { of time practiced DBT had } \\
\text { a moderate correlation } \\
\text { with DBT knowledge }\end{array}$ \\
\hline & & & Other papers & & \\
\hline $\begin{array}{l}\text { James et al., } \\
2015[60]\end{array}$ & $\begin{array}{l}\text { America. Psychiatric } \\
\text { facility }\end{array}$ & $\begin{array}{l}\text { Trial process } \\
\text { analysis }\end{array}$ & $\begin{array}{l}\text { Service embedded } \\
\text { repeated measures } \\
\text { evaluation }\end{array}$ & Good outcomes & $\begin{array}{l}\text { Grant funded participants } \\
\text { had higher attrition }\end{array}$ \\
\hline $\begin{array}{l}\text { Kinsey \& Reed, } \\
2015 \text { [43] }\end{array}$ & $\begin{array}{l}\text { America. Native American } \\
\text { tribe outpatient mental } \\
\text { health and substance use } \\
\text { service }\end{array}$ & $\begin{array}{l}\text { Programme } \\
\text { description }\end{array}$ & N/A & N/A & $\begin{array}{l}\text { Programme had run for } 14 \\
\text { years and had a good } \\
\text { relationship with the tribal } \\
\text { community }\end{array}$ \\
\hline $\begin{array}{l}\text { Baillie \& Slater, } \\
2014 \text { [39] }\end{array}$ & $\begin{array}{l}\text { UK. Community } \\
\text { intellectual } \\
\text { disability service }\end{array}$ & $\begin{array}{l}\text { Programme } \\
\text { description }\end{array}$ & Mostly discussion & $\begin{array}{l}\text { Some evidence that } \\
\text { patients developed } \\
\text { emotion regulation and } \\
\text { distress tolerance skills }\end{array}$ & $\begin{array}{l}\text { DBT service had been in } \\
\text { operation for four years }\end{array}$ \\
\hline $\begin{array}{l}\text { Engle et al., } \\
2013 \text { [42] }\end{array}$ & $\begin{array}{l}\text { America. College } \\
\text { counselling service }\end{array}$ & $\begin{array}{l}\text { Programme } \\
\text { description }\end{array}$ & Between groups & $\begin{array}{l}\text { Reduced psychiatric } \\
\text { and substance use } \\
\text { hospitalisations. Reduced } \\
\text { college absence due to } \\
\text { mental health problems }\end{array}$ & $\begin{array}{l}\text { Team received intensive } \\
\text { training. Carried caseloads } \\
\text { of up to seven patients } \\
\text { plus one skills group }\end{array}$ \\
\hline $\begin{array}{l}\text { Arroyo et al., } \\
2012[38]\end{array}$ & $\begin{array}{l}\text { America. Mount Sinai } \\
\text { East Harlem health } \\
\text { outreach project }\end{array}$ & $\begin{array}{l}\text { Programme } \\
\text { description }\end{array}$ & N/A & $\begin{array}{l}\text { Anecdotal evidence of } \\
\text { patient improvement }\end{array}$ & $\begin{array}{l}\text { Implemented skills group } \\
\text { only. Therapists received } \\
\text { fortnightly supervision }\end{array}$ \\
\hline $\begin{array}{l}\text { Lajoie et al., } \\
2011[44]\end{array}$ & $\begin{array}{l}\text { America. Residency } \\
\text { run clinic }\end{array}$ & $\begin{array}{l}\text { Programme } \\
\text { description }\end{array}$ & N/A & N/A & $\begin{array}{l}\text { Implemented all core } \\
\text { DBT components }\end{array}$ \\
\hline $\begin{array}{l}\text { Morrissey \& } \\
\text { Ingamells, } \\
2011[47]\end{array}$ & $\begin{array}{l}\text { UK. Learning disability } \\
\text { forensic secure service }\end{array}$ & $\begin{array}{l}\text { Programme } \\
\text { description }\end{array}$ & $\begin{array}{l}\text { Naturalistic outcomes } \\
\text { reported }\end{array}$ & $\begin{array}{l}\text { Reduced symptoms } \\
\text { and distress. Reduced } \\
\text { perceived risk }\end{array}$ & $\begin{array}{l}\text { Implemented programme } \\
\text { over six years }\end{array}$ \\
\hline $\begin{array}{l}\text { Pasieczny \& } \\
\text { Connor, } 2011 \text { [66] }\end{array}$ & $\begin{array}{l}\text { Australia. Adolescent } \\
\text { mental health service }\end{array}$ & $\begin{array}{l}\text { Trial process } \\
\text { analysis }\end{array}$ & Between groups & $\begin{array}{l}\text { Patients of intensively } \\
\text { trained therapists had } \\
\text { better outcomes in terms } \\
\text { of DSH and suicide } \\
\text { attempts }\end{array}$ & $\begin{array}{l}\text { Therapists worked in DBT } \\
\text { team part-time. Therapist } \\
\text { adherence ranged } \\
\text { nine-to-12 (maximum } \\
\text { achievable }=12 \text { ) }\end{array}$ \\
\hline Little et al., 2010 [46] & $\begin{array}{l}\text { America. Residential } \\
\text { service }\end{array}$ & $\begin{array}{l}\text { Programme } \\
\text { description }\end{array}$ & N/A & $\begin{array}{l}\text { Self-reported patient } \\
\text { improvement and } \\
\text { positive feedback }\end{array}$ & $\begin{array}{l}\text { DBT was the best } \\
\text { implemented treatment in } \\
\text { the service; had furthest } \\
\text { reach, most staff support } \\
\text { and needed less senior } \\
\text { administrative support. } \\
\text { Minimal attrition }\end{array}$ \\
\hline $\begin{array}{l}\text { Sampl et al., } 2010 \\
\text { [48] }\end{array}$ & $\begin{array}{l}\text { America. } \\
\text { Correctional setting }\end{array}$ & $\begin{array}{l}\text { Programme } \\
\text { description }\end{array}$ & N/A & N/A & $\begin{array}{l}\text { Primarily just implemented } \\
\text { skills group }\end{array}$ \\
\hline $\begin{array}{l}\text { Blennerhassett } \\
\text { et al., } 2009 \text { [54] }\end{array}$ & $\begin{array}{l}\text { Ireland. Community } \\
\text { mental health team }\end{array}$ & $\begin{array}{l}\text { Trial process } \\
\text { paper }\end{array}$ & Repeated measures & $\begin{array}{l}\text { Improved risks, symptoms, } \\
\text { functioning and subjective } \\
\text { wellbeing. Reduced } \\
\text { hospitalisations and } \\
\text { reduced costs }\end{array}$ & $\begin{array}{l}\text { Therapists completed } \\
\text { intensive training but DBT } \\
\text { team not established in } \\
\text { the service }\end{array}$ \\
\hline
\end{tabular}


Table 2 Implementation papers, programme descriptions and trial process analysis papers (Continued)

\begin{tabular}{|c|c|c|c|}
\hline Reference & Country \& service context & Paper type & Methodology \\
\hline Kerr et al., 2009 [62] & $\begin{array}{l}\text { America. Low resourced } \\
\text { rural training clinic }\end{array}$ & $\begin{array}{l}\text { Trial process } \\
\text { analysis }\end{array}$ & Case study \\
\hline $\begin{array}{l}\text { Hjalmarsson } \\
\text { et al., } 2008 \text { [59] }\end{array}$ & $\begin{array}{l}\text { Sweden. Outpatient } \\
\text { services }\end{array}$ & $\begin{array}{l}\text { Trial process } \\
\text { analysis }\end{array}$ & Repeated measures \\
\hline
\end{tabular}

et al., 2008 [59] services analysis

Woodberry \& Popenoe, $2008[71]$

America. Adolescent and family outpatient clinic

Trial process analysis

Repeated measures

DBT outcomes

There were "meaningful"
changes in suicidality
and misery ratings
and misery ratings

Patients had reduced para-suicidal behaviours and psychological distress

Good outcomes reported

Good outcomes reported

\section{inter} received less intensive or in-service training. The hospital provided some money to support staff training
Comtois et al 2007 [57]

Zinkler et al., 2007 [52]

Brassington
\& Krawitz,

2006 [56]

Koons et al., 2006 [65]

Lew et al. 2006 [45]

Nelson-Gray et al., 2006 [64]

Vitacco \& Van Rybroek, 2006 [50]
America. Harbour view mental health servicescommunity mental

health centre

Australia, Adult mental Trial process health outpatient service analysis

UK. Newham project for BPD

New Zealand. Mental health service

America. Division of vocational rehabilitation

America. Intellectual disability service

America. Outpatient adolescent clinic

America. Forensic hospitals analysis

Programme
description description

Pilot trial process analysis

Trial process analysis

Programme description

Trial process analysis
Trial process Repeated measures

N/A

Provided service outcome data

Repeated measures patients completed the hospitalisations. Improved
Reduced DSH, A\&E visits and inpatient admissions

Reduced depression and frequency of suicide attempts and patient functioning and reduced intervention duration

Reduced hospitalisation and DSH frequency

Noted DBT staff were highly trained. Implemented all DBT components and incorporated access to DBT relevant services

The team comprised 12 therapists. Attrition was 31\%

Annual service cost $£ 92,000$. Therapists worked part-time on DBT team. Staff satisfaction and retention high

Good outcomes reported

Implementation reportedly successful. Team staffed by part-time therapists and at the end of the trial team had a dedicated budget

At six months improved depression, hopelessness, anger expression, work role satisfaction and number of hours worked

Provided just DBT skills group

Eight learning disability programme. DSH gradually reduced

Reduced negative behaviours, externalising and internalising symptoms, and depression. Increased positive behaviours

Staff carried caseloads of eight. Parents and staff also attended the skills groups

Trained a high number of graduate students and these students achieved 88\% intervention delivery fidelity over eight groups

N/A

N/A 
Table 2 Implementation papers, programme descriptions and trial process analysis papers (Continued)

\begin{tabular}{|c|c|c|c|c|c|}
\hline Reference & Country \& service context & Paper type & Methodology & DBT outcomes & $\begin{array}{l}\text { Implementation } \\
\text { relevant outcomes }\end{array}$ \\
\hline $\begin{array}{l}\text { Nee \& Farman, } \\
2005 \text { [63] }\end{array}$ & UK. Female prisons & $\begin{array}{l}\text { Trial process } \\
\text { analysis }\end{array}$ & $\begin{array}{l}\text { Between groups (with a } \\
\text { waiting list control) }\end{array}$ & $\begin{array}{l}\text { The majority of } \\
\text { completers showed } \\
\text { overall improvement } \\
\text { with notable effect sizes }\end{array}$ & $\begin{array}{l}\text { Implementation problems } \\
\text { believed to contribute to } \\
\text { high attrition }\end{array}$ \\
\hline $\begin{array}{l}\text { APA Gold } \\
\text { Award, } 2004 \text { [37] }\end{array}$ & $\begin{array}{l}\text { America. Grove street } \\
\text { adolescence } \\
\text { residence- residential } \\
\text { care service }\end{array}$ & $\begin{array}{l}\text { Programme } \\
\text { description }\end{array}$ & N/A & $\begin{array}{l}\text { Outcome data indicated } \\
\text { the programme was } \\
\text { effective }\end{array}$ & $\begin{array}{l}\text { Provided all DBT } \\
\text { components and had } 18.7 \\
\text { full time equivalent staff } \\
\text { members }\end{array}$ \\
\hline $\begin{array}{l}\text { Ben-Porath } \\
\text { et al., } 2004 \text { [53] }\end{array}$ & $\begin{array}{l}\text { America. Urban } \\
\text { community mental } \\
\text { health centre }\end{array}$ & $\begin{array}{l}\text { Trial process } \\
\text { analysis }\end{array}$ & Repeated measures & $\begin{array}{l}\text { Reduced life threatening, } \\
\text { therapy interfering and } \\
\text { QOL interfering } \\
\text { behaviours }\end{array}$ & $\begin{array}{l}\text { Implemented all core DBT } \\
\text { components. Three of the } \\
\text { eight DBT team members } \\
\text { left within six months }\end{array}$ \\
\hline $\begin{array}{l}\text { Katz et al., } \\
2004 \text { [61] }\end{array}$ & $\begin{array}{l}\text { Canada. Adolescent } \\
\text { inpatient service }\end{array}$ & $\begin{array}{l}\text { Pilot trial } \\
\text { process } \\
\text { analysis }\end{array}$ & Between groups & $\begin{array}{l}\text { Reduced behavioural } \\
\text { incidents on ward. } \\
\text { Equivalent to TAU in } \\
\text { reducing para-suicidal } \\
\text { behaviour, depression } \\
\text { symptoms and suicidal } \\
\text { ideation at one year } \\
\text { follow-up }\end{array}$ & $\begin{array}{l}\text { Provided skills group, } \\
\text { individual therapy and } \\
\text { milieu therapy }\end{array}$ \\
\hline Sunseri, 2004 [49] & $\begin{array}{l}\text { America. Residential } \\
\text { centre for adolescents }\end{array}$ & $\begin{array}{l}\text { Programme } \\
\text { description }\end{array}$ & $\begin{array}{l}\text { Naturalistic } \\
\text { outcomes reported }\end{array}$ & $\begin{array}{l}\text { Reduced attrition, } \\
\text { inpatient days and } \\
\text { duration of restraint } \\
\text { and seclusion }\end{array}$ & $\begin{array}{l}\text { Staff confidence grew } \\
\text { with DBT implementation }\end{array}$ \\
\hline $\begin{array}{l}\text { Eccleston \& Sobello, } \\
2002[58]\end{array}$ & Australia. Prison service & $\begin{array}{l}\text { Pilot trial } \\
\text { process } \\
\text { analysis }\end{array}$ & Repeated measures & $\begin{array}{l}\text { Trend improvement } \\
\text { supported by patient } \\
\text { feedback }\end{array}$ & $\begin{array}{l}\text { Anecdotally, a range } \\
\text { of staff saw programme } \\
\text { benefits }\end{array}$ \\
\hline $\begin{array}{l}\text { Rathus \& Miller, } \\
2002 \text { [68] }\end{array}$ & $\begin{array}{l}\text { America. Adolescent } \\
\text { outpatient clinic }\end{array}$ & $\begin{array}{l}\text { Trial process } \\
\text { analysis }\end{array}$ & Between groups & $\begin{array}{l}\text { Reduced hospitalisations } \\
\text { and increased retention } \\
\text { but did not reduce suicide } \\
\text { attempts }\end{array}$ & $\begin{array}{l}\text { DBT transportable to } \\
\text { real-world settings: } \\
\text { provided in a hospital, not } \\
\text { a university-based clinic }\end{array}$ \\
\hline $\begin{array}{l}\text { Trupin et al., } \\
2002 \text { [69] }\end{array}$ & $\begin{array}{l}\text { America. Incarceration } \\
\text { centre for female } \\
\text { juvenile offenders }\end{array}$ & $\begin{array}{l}\text { Trial process } \\
\text { analysis }\end{array}$ & Between groups & $\begin{array}{l}\text { Only one unit showed } \\
\text { reduced behaviour } \\
\text { problems }\end{array}$ & $\begin{array}{l}\text { Only one unit showed less } \\
\text { staff use of punitive } \\
\text { responses. Not all staff } \\
\text { adherent to DBT }\end{array}$ \\
\hline $\begin{array}{l}\text { van den Bosch } \\
\text { et al., } 2002 \text { [70] }\end{array}$ & $\begin{array}{l}\text { Netherlands. Addiction } \\
\text { treatment centre }\end{array}$ & $\begin{array}{l}\text { Trial process } \\
\text { analysis }\end{array}$ & $\begin{array}{l}\text { Randomised } \\
\text { controlled trial }\end{array}$ & $\begin{array}{l}\text { Reduced DSH but did not } \\
\text { improve substance use }\end{array}$ & $\begin{array}{l}\text { Over time therapists said } \\
\text { they felt less isolated, more } \\
\text { competent and } \\
\text { experienced more work } \\
\text { satisfaction. Consultation } \\
\text { team attendance } 100 \% \text {. } \\
\text { Attrition } 37 \%\end{array}$ \\
\hline $\begin{array}{l}\text { Bohus et al., } \\
2000[55]\end{array}$ & $\begin{array}{l}\text { Germany. Inpatient } \\
\text { service }\end{array}$ & $\begin{array}{l}\text { Pilot trial } \\
\text { process } \\
\text { analysis }\end{array}$ & Repeated measures & $\begin{array}{l}\text { Reduced DSH, } \\
\text { disassociation } \\
\text { phenomena and } \\
\text { depressive symptoms }\end{array}$ & $\begin{array}{l}\text { Intervention was rated } \\
\text { positively by staff and } \\
\text { patients and this was an } \\
\text { impetus to conduct the trial }\end{array}$ \\
\hline $\begin{array}{l}\text { Wolpow et al., } \\
2000 \text { [51] }\end{array}$ & $\begin{array}{l}\text { America. Residential } \\
\text { programme }\end{array}$ & $\begin{array}{l}\text { Programme } \\
\text { description }\end{array}$ & $\begin{array}{l}\text { Included a service } \\
\text { evaluation }\end{array}$ & $\begin{array}{l}\text { Patients gave positive } \\
\text { feedback and } \\
\text { observations were } \\
\text { positive }\end{array}$ & $\begin{array}{l}\text { Residential staff became } \\
\text { more positive about DBT }\end{array}$ \\
\hline $\begin{array}{l}\text { Gold Award, } \\
1998 \text { [41] }\end{array}$ & $\begin{array}{l}\text { America. Mental health } \\
\text { centre }\end{array}$ & $\begin{array}{l}\text { Programme } \\
\text { description }\end{array}$ & N/A & $\begin{array}{l}\text { Positive patient outcomes } \\
\text { and reduced costs } \\
\text { reported }\end{array}$ & $\begin{array}{l}13 \text { staff in DBT team. } \\
\text { Provided all DBT } \\
\text { components plus } \\
\text { additional DBT related } \\
\text { services. Team funding the } \\
\text { equivalent of } £ 520,000 \text { per } \\
\text { annum }\end{array}$ \\
\hline $\begin{array}{l}\text { Barley et al., } 1993 \\
\text { [40] }\end{array}$ & $\begin{array}{l}\text { America. Inpatient } \\
\text { psychiatric hospital }\end{array}$ & $\begin{array}{l}\text { Programme } \\
\text { description }\end{array}$ & $\begin{array}{l}\text { Naturalistic } \\
\text { outcome evaluation }\end{array}$ & $\begin{array}{l}\text { Reduced para-suicidal } \\
\text { behaviour }\end{array}$ & $\begin{array}{l}\text { Transitioned to a DBT } \\
\text { model over a two year } \\
\text { period }\end{array}$ \\
\hline
\end{tabular}


as, smaller caseloads and enabling staff to hold a highly visible role in the service seemed more effective (e.g. [40]) than providing tangible reinforcements (e.g. [65]) although see [48] for an exception.

\section{System interventions}

In the US services need to ensure they receive sufficient referrals to remain viable and so coordination with external agencies is necessary [36]. There were five examples of coalitions facilitating implementation $([14,42,54,55,59])$. One research group suggested that training courses and merging consultation teams might foster coalitions [23] and there was an example of a service establishing two consultation teams: one service-led, the other interagency [45].

\section{Evidence}

Informed by PARIHS our initial framework referred to the sub-elements of research and published guidance, clinical experience and professional knowledge, preferences and experiences and local knowledge. However, our search yielded just two primary sub-elements (preferences and experiences and local knowledge and evaluation):

\section{Preferences and experiences}

Some therapists expressed a preference for DBT [28] but $47 \%$ of therapists said there were challenges in recruiting sufficient patients [23]. Patients reported that they liked many aspects of DBT [29], though they need sufficient cognitive capacity to understand DBT skills and this may constitute a barrier for some [25]. The literature contained evidence that recruitment (e.g. [38]) and attrition (e.g. [52]) could be a problem and there were many attempts to reduce attrition including: ensuring participation was voluntary (e.g. [51]), careful selection of patients (e.g. [42]), providing more information about what DBT would entail (e.g. [53]) and, when appropriate, involving caregivers (e.g. [46]). On two occasions tangible reinforcement was offered [40,64].

\section{Local knowledge and evaluation}

Evidence of clinical improvement can reinforce implementation attempts [24], although only $7 \%$ of respondents in one survey agreed that improved patient outcomes were an implementation facilitator [31]. Sometimes demonstrating good patient outcomes generated interest in DBT [37] and led to further funding [43]. However, there were only five examples of services routinely evaluating outcomes ([37, 39, 42, 47, 48]).

\section{Facilitation}

Our initial framework referenced six sub-elements (strategies, support, training, coaching and ongoing consultation, facilitator skills/ qualities and recruitment and selection). However, our search and synthesis yielded two primary sub-elements (team capacity and commitment, and training and ongoing support):

\section{Team capacity and commitment}

Some therapists thought the effectiveness of DBT was solely due to its techniques and theory [29], but this view was not universal. Several optimal therapist attributes were detailed including a stance of equality, an ability to synthesise validation and challenge, a good understanding of DBT skills, as well as, group management and teaching abilities [25]. Therapist confidence also seemed important [27] and this could be enhanced through DBT implementation [24, 28] and training [35]. Administrators selected staff based on their seniority and motivation and recruited to ensure team diversity [36]. Therapists' academic qualifications seem less important [26], but they do need to be skilled clinicians [31].

Insufficient staffing can jeopardise sustainability $([23,30,36])$ and staff turnover is a further barrier $[23,31]$. For instance, in one prospective implementation study $55 \%$ of therapists remained working at their original organisation at two year follow-up [35]. A possible reason for retention problems is that new DBT therapists initially reported increased stress levels and there was a tendency (although this was not statistically significant) for staff burnout to occur over time [28]. A small association suggested that larger teams implement more DBT components [26]. Smaller teams are likely to operate within larger services, with staff having additional roles. These nested programmes seem common as several therapists reported working in the DBT team part-time [28] and contrary to the Ditty et al. [26] findings, there were examples of successful nested teams (e.g. [56]) and teams dependent on part-time staffing (e.g. [52]).

\section{Training and ongoing support}

Clinicians from diverse disciplines can acquire a solid grounding in DBT through training [34]. Training facilitates implementation [31] and attending more training is associated with greater confidence and use of DBT [27]. For instance, training significantly increased the use of skills training, treatment targets, daily diary cards and dialectic strategies [35]. DBT knowledge also moderately correlated with all indices of training [34]. Unfortunately, limited feedback about training has been collected. Therapists reported that training enabled them to use DBT in their practice but they wanted more detailed instruction on how to perform specific interventions, such as conducting chain analysis of problem behaviour [23]. In one study, E-learning was most successful in increasing reported application of DBT [32]. In an earlier report, instructor-led training improved therapist 
self-efficacy and satisfaction but no method increased therapist skilfulness [33]. There was some evidence that training could improve clinical outcomes (e.g. [66]) but a lack of training was not always a barrier: graduate students with minimal training achieved $88 \%$ fidelity with DBT methods when facilitating skills groups [64].

On-going consultation is important [23,36] and lacking access to a DBT consultation team can be an implementation barrier [27]. DBT consultation teams can help therapists achieve dialectical synthesis [25] and complement $[25,28]$ or compensate for lack of training [34]. Access to individual supervision is also important [26]: lack of supervision was the most frequently reported barrier to using DBT skills in one report [33] and in a UK survey [31], 34\% of respondents said supervision facilitated the use of DBT. Limited feedback has been collected about supervision experiences: therapists reported that supervision increases both stress and coping [28].

\section{DBT}

Our initial framework identified four sub-elements related to the intervention (design quality and packaging, adaptability, complexity and costs). Our search and synthesis yielded sub-elements related to the design quality, packaging and costs:

\section{Design quality, packaging and costs}

DBT can be a complex therapy to implement: several DBT skills can be difficult to understand and apply [25] and trainers have reported that therapists have difficulty applying DBT's behavioural components [34]. Aspects of DBT which seem important are its treatment contract emphasising shared responsibility [29] and its adaptability [24, 30]. For instance, despite some authors believing that DBT's manual-based nature is important [29, 34], there were many examples of adaptations (e.g. [48]) with adjustments often altering how telephone skills coaching was provided (e.g. [67]). In the US, limited reimbursement is a barrier to implementing DBT $[27,36]$ and in the UK, $29 \%$ of survey respondents said that allocating sufficient finances to DBT delivery was an implementation facilitator [31].

\section{Implementation process}

CFIR separates the implementation process into sub-elements related to execution, engagement, planning, evaluation and reflection. It was not possible to dissect these individual components in the literature. However, there were two examples of clearly executed implementation plans $[40,46]$ and five examples of services forming teams to oversee the implementation process $([41,49,51,57,59])$. A lack of an implementation plan can be an implementation barrier [31] but plans do not guarantee success. For instance, one study planned to introduce a number of resources (e.g., demonstration videos, an online forum and telephone consultation) to improve DBT adherence during implementation [23]. During the study there were no requests for consultation and in post-implementation interviews therapists did not refer to any of the available resources. This study highlights that providing resources alone is unlikely to promote implementation.

\section{Discussion}

This critical literature review synthesised the DBT implementation literature to refine a DBT implementation framework. The framework sufficiently captured the data and no new elements or sub-elements were required (see Additional file 4). However, some refinements were made to create a more parsimonious and relevant framework for DBT. For instance, coding indicated that some sub-elements were capturing similar data. For example, the sub-elements 'individual characteristics', 'facilitator skills/ qualities', and 'recruitment and selection' were re-conceived into a sub-element called team capacity and commitment. Additionally, some sub-elements arose infrequently in the literature (e.g., research and published guidance) and these were therefore omitted (the refined framework is illustrated in Fig. 3). However, we acknowledge that limited literature on an implementation barrier is not necessarily evidence that the barrier is not significant in DBT. For instance, cost may prevent both implementation and research meaning that the magnitude of barriers related to cost may not be sufficiently reflected in the framework, as the literature primarily reflects successfully funded work.

The utility of transdisciplinary implementation frameworks, such as PARIHS [5, 6], is highlighted by these findings: elements primarily derived from existing frameworks effectively captured DBT implementation barriers and facilitators. The critical review process also proved to be useful in guiding the framework refinement and the synthesis of the literature. However, only 14 papers were retrieved that specifically focused on DBT implementation and this suggests that a DBT implementation framework may usefully underscore the most important considerations for DBT implementers.

The DBT implementation framework is a useful resource for DBT practitioners and service leaders who are planning (or overseeing current) DBT implementations. The synthesis indicates that implementers should consider the following recommendations:

- When introducing DBT into practice clinicians and organisations should encourage the staff team to operate a benign approach to BPD and ensure there are good communication systems in place.

- When establishing a DBT team, it seems important to recruit therapists with sufficient cognitive 


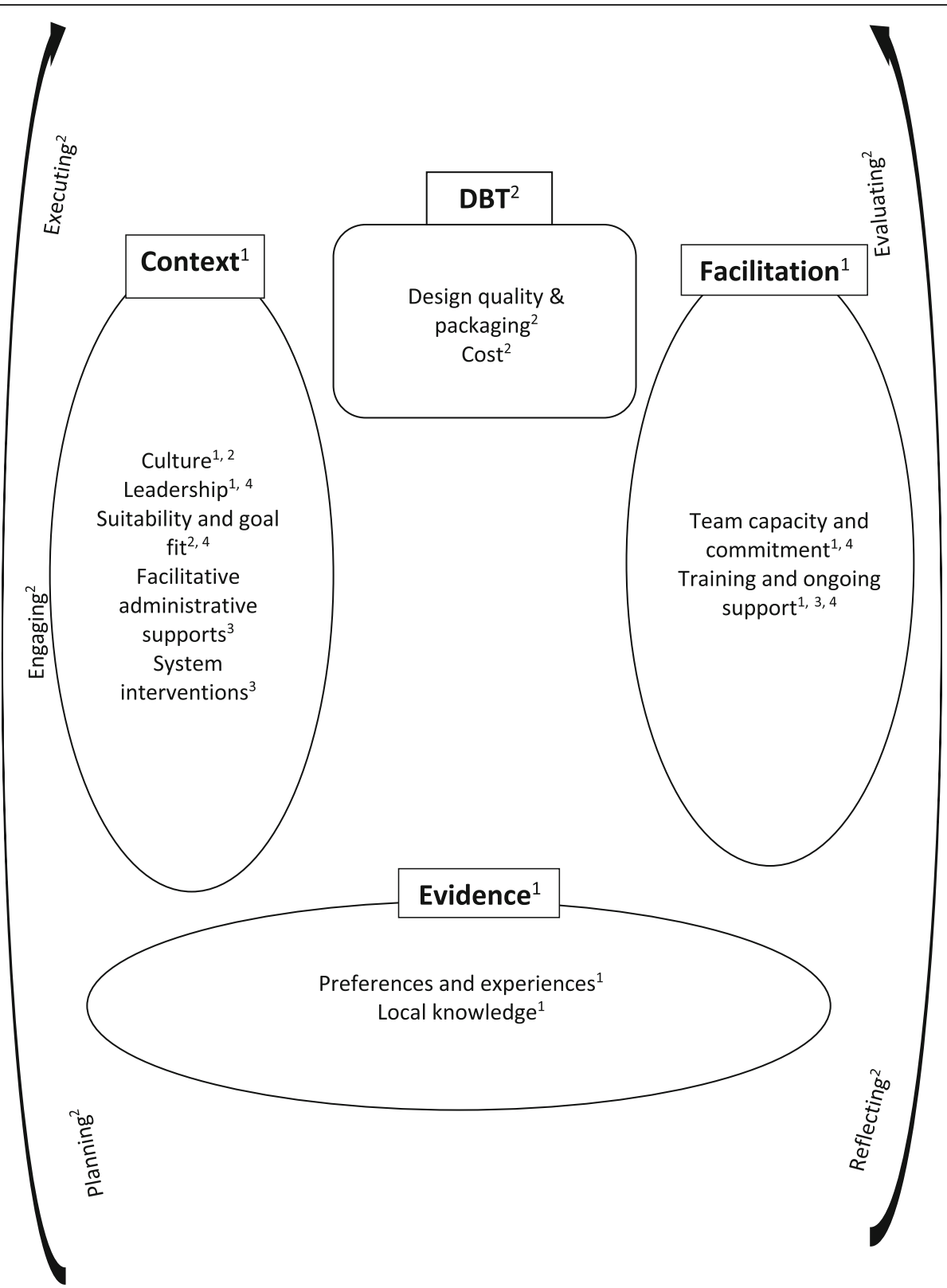

Fig. 3 Revised DBT implementation framework. Key: PARIHS, 2004'; Damschroder et al., 2009²; Fixsen \& Blasé, 2009³, Swales 2010a, 2010b

flexibility, whose personal qualities align with those espoused by DBT, such as, having a nonjudgemental stance.

- The DBT team will benefit from on-going supervision and consultation and therapists should receive adequate training.

- Leadership support is beneficial and in situations where implementation is not pre-planned, a DBT champion can help.

- It is beneficial for services to evaluate whether DBT needs adapting to suit their organisation.
Despite the apparent strengths of the DBT implementation framework, the limitations of the literature need to be taken into account. The framework's generalisability cannot be ascertained as the reviewed literature only provided information about implementation in Western contexts and primarily reported on implementation in statutory outpatient mental health services. The most commonly retrieved papers were trial reports and implementing DBT in a research context may have significant differences from implementation in clinical services. Furthermore, the decision to include only published literature biased the review 
towards considering effective DBT implementations as most trials and programme descriptions reported positive results. In particular the literature reviewed, with the exception of a DBT implementation with Native Americans [43], cannot inform how DBT implementation is achieved with marginalised and particularly high risk populations, such as cultural minority groups. When more information about DBT implementations with such populations become available, the framework may require refinement.

The implementation papers reviewed also had methodological limitations. Most data was collected retrospectively and relied on self-reports of implementation success (e.g. [26]). Samples may not have been representative, for instance, the response rate in one study was approximately 14\% [30]. Furthermore, survey instruments had not always been validated (e.g. [35]) and most quantitative data was correlational (e.g. [26]), so causation could not be inferred.

Limitations in the literature and framework provide opportunities for future research. It is acknowledged that interrater agreement when using the framework to code data could be improved. The current framework is sufficiently detailed for use by DBT practitioners and service leaders who are planning implementation, but in a research and academic context one next step will be to develop more precise definitions of some sub-elements. Although, the current literature cannot inform how implementation barriers and facilitators interact or how they are weighted in different contexts, a few tentative potential relationships warrant further exploration. For example, communication and contingency management might be particularly important in organisations providing team approaches, such as, inpatient services. Access to on-going support may be particularly important if staff have not received comprehensive DBT training. The complexity of DBT may only form a barrier if clients and staff have not been appropriately selected. To refine and further develop the DBT implementation framework in the academic context, another next step will be to undertake further research to explore these tentative ideas about how the framework elements interact and are weighted. To explore these relationships further, prospectively collected data will be needed as is planned in the EMPOWER research programme (NIHR Programme Grant: RP-PG-1212-20,011).

\section{Conclusions}

This review has explored the DBT implementation literature and developed a bespoke framework to inform future implementations. The literature synthesis has highlighted some important implementation considerations but prospective DBT implementation studies are now needed to explore the relative weighting of and relationships amongst these barriers and facilitators.

\section{Additional files}

\begin{abstract}
Additional file 1: A detailed description of the DBT implementation framework. Describes the DBT implementation framework and its elements in greater detail. (DOCX $22 \mathrm{~kb}$ )
\end{abstract}

Additional file 2: Critical literature review: example search. Provides the literature search used in the CINAHL (EBSCO) database. (DOCX $12 \mathrm{~kb}$ )

Additional file 3: Data extraction template. The data extraction form used in the critical literature review. (DOCX $12 \mathrm{~kb}$ )

Additional file 4: DBT implementation framework: overview of assigned codes. Illustrates how framework elements and sub-elements were operationalised into codes. Lists which codes were assigned by paper and study type. Also provides a tally of how many times each code was assigned. (DOCX $30 \mathrm{~kb}$ )

\section{Abbreviations}

BPD: Borderline Personality Disorder; CFIR: Consolidated Framework for Implementation Research; DBT: Dialectical Behaviour Therapy; DBTSE: Dialectical Behaviour Therapy- Skills for Employment; EMPOWER: Enabling and Motivating people (with a Personality Disorder) in Occupation, Wellbeing, Education and Responsibility; PARIHS: Promoting Action on Research Implementation in Health Services

\section{Acknowledgements}

We would like to thank Rhiannon Whittaker and Stephen Stansfeld for their comments on an earlier draft of the paper.

\section{Funding}

EMPOWER (Enabling and Motivating people (with a Personality Disorder) in Occupation, Wellbeing, Education and Responsibility) is a NIHR funded programme (Programme Grant no RP-PG-1212-20011). The funder had no role in conduct or analysis of the critical literature review or the decision to submit the review for publication. The views expressed are those of the authors and not necessarily those of the NHS, the NIHR or the Department of Health.

Availability of data and materials

Not applicable as no new data collected or materials created.

\section{Authors' contributions}

GT conducted the critical literature review, drafted and revised the paper. JRM designed the framework and assisted in the revision of the paper. MS designed the DBT implementation framework and assisted in the revision of the paper. LW designed the DBT implementation framework, assisted with the critical literature review and revised the paper. JF designed the EMPOWER study and assisted in the revision of the paper. All authors read and approved the final manuscript.

\section{Author information}

GT: Was a Research Assistant on the EMPOWER project and has a background in clinical psychology.

JRM: Is the Pro-Vice Chancellor for Research and Impact at Bangor University. She developed the PARIHS implementation framework and is an established researcher in implementation science.

MS: Is one of the founders of the British Isles Dialectical Behaviour Therapy Training company. She is a Senior Lecturer in Clinical Psychology at Bangor University and works for Betsi Cadwaladr University Health Board. LW: Is Senior Lecturer in the School of Healthcare Sciences at Bangor University. She has a research interest in implementation science. JF: Is a Chartered Consultant Clinical Psychologist with North East London Foundation Trust and a Senior Lecturer in Clinical Psychology. She is the Clinical Lead for the Intensive Management of Personality disorder: Assessment and Recovery Team (IMPART) and is the designer and Chief Investigator of the EMPOWER study.

\section{Ethics approval and consent to participate} N/A

\section{Consent for publication} N/A 


\section{Competing interests}

There are no financial competing interests. MS and JF are members of DBT Training British Isles.

\section{Publisher's Note}

Springer Nature remains neutral with regard to jurisdictional claims in published maps and institutional affiliations.

\section{Author details \\ 'Gill Toms, School of Healthcare Sciences, Bangor University, Fron Heulog, Bangor, Gwynedd LL57 2EF, UK. ${ }^{2}$ North Wales Clinical Psychology Programme, School of Psychology, Brigantia Building, Bangor University, and Health Psychology, University College London, Gower Street, London WC1E 6BT, UK \\ Received: 7 November 2018 Accepted: 5 February 2019 \\ Published online: 12 February 2019} Bangor, Gwynedd LL57 2DG, UK. ${ }^{3}$ Research Department of Clinical, Education

\section{References}

1. Linehan M. Cognitive-behavioral treatment of borderline personality disorder. New York: Guilford Press; 1993

2. Valentine SE, Bankoff SM, Poulin RM, Reidler EB, Pantalone DW. The use of dialectical behavioral therapy skills training as stand-alone treatment: a systematic review of the treatment outcome literature. J Clin Psychol. 2015. https://doi.org/10.1002/jclp.22114.

3. Bendit N. Reputation and science: examining the effectiveness of DBT in the treatment of borderline personality disorder. Australas Psychiatry. 2014. https://doi.org/10.1177/1039856213510959.

4. Panos PT, Jackson JW, Hasan O, Panos A. Meta-analysis and systematic review assessment of the efficacy of dialectical behavior therapy (DBT). Res Soc Work Pract. 2014. https://doi.org/10.1177/1049731513503047.

5. Kitson A, Harvey G, McCormack B. Enabling the implementation of evidence based practice: a conceptual framework. Qual Health Care. 1998. https://doi. org/10.1136/qshc.7.3.149.

6. Rycroft-Malone J. The PARIHS framework-a framework for quiding the implementation of evidence-based practice. J Nurs Care Qual. 2004. https:// doi.org/10.1097/00001786-200410000-00002

7. Damschroder LJ, Aron DC, Keith RE, Kirsch SR, Alexander JA, Lowery JC. Fostering implementation of health services research findings into practice: a consolidated framework for advancing implementation science. Implement Sci. 2009. https://doi.org/10.1186/1748-5908-4-50.

8. Fixsen DL, Naoom SF, Blase KA, Friedman RM, Wallace F. Implementation research: a synthesis of the literature. Tampa FL, America: University of South Florida, Louis de la parte Florida mental health institute, The National Implementation Research Network (FMHI publication \#231); 2005.

9. Fixsen DL, Blase KA, Naoom SF, Wallace F. Core implementation components. Res Soc Work Pract. 2009. https://doi.org/10.1177/ 1049731509335549

10. National Collaborating Centre for Mental Health. The improving access to psychological therapies manual. UK: NCCMH; 2018.

11. Swales MA. Implementing DBT: selecting, training and supervising a team. Cognitive Behaviour Therapist. 2010a. https://doi.org/10.1017/ S1754470X10000061.

12. Swales MA. Implementing dialectical behaviour therapy: organizational pretreatment. Cognitive Behaviour Therapist. 2010b. https://doi.org/10.1017/ S1754470X10000115

13. Grant MJ, Booth A. A typology of reviews: an analysis of 14 review types and associated methodologies. Health Inf Libr J. 2009. https://doi.org/10. 1111/j.1471-1842.2009.00848.x.

14. Berzins LG, Trestman RL. The development and implementation of dialectical behavior therapy in forensic settings. Int J Forensic Ment Health. 2004. https://doi.org/10.1080/14999013.2004.10471199.

15. Burroughs T, Somerville J. Utilization of evidenced based dialectical behavioral therapy in assertive community treatment: examining feasibility and challenges. Community Ment Health J. 2013. https://doi.org/10.1007/ s10597-012-9485-2.

16. Chugani CD. Dialectical behavior therapy in college counseling centers: current literature and implications for practice. J Coll Stud Psychother. 2015. https://doi.org/10.1080/87568225.2015.1008368.
17. Huffman JC, Stern TA, Harley RM, Lundy NA. The use of DBT skills in the treatment of difficult patients in the general hospital. Psychosomatics. 2003. https://doi.org/10.1176/appi.psy.44.5.421.

18. Koerner K. What must you know and do to get good outcomes with DBT? Behav Ther. 2013. https://doi.org/10.1016/j.beth.2013.03.005.

19. McHugh RK, Barlow DH. The dissemination and implementation of evidence-based psychological treatments: a review of current efforts. Am Psychol. 2010. https://doi.org/10.1037/a0018121.

20. Scheel KR. The empirical basis of dialectical behavior therapy: summary, critique and implications. Clin Psychol. 2000. https://doi.org/10.1093/ clipsy.7.1.68.

21. Swenson CR, Torrey WC, Koerner K. Implementing dialectical behavior therapy. Psychiatr Serv. 2002. https://doi.org/10.1176/appi.ps.53.2.171.

22. Swenson CR. How can we account for DBT's widespread popularity? Clin Psychol. 2000. https://doi.org/10.1093/clipsy.7.1.87.

23. Carmel A, Rose M, Fruzzetti AE. Barriers and solutions to implementing dialectical behavior therapy in a public behavioral health system. Admin Pol Ment Health. 2014. https://doi.org/10.1007/s10488-013-0504-6.

24. Chwalek CM, McKinney CH. The use of dialectical behavior therapy (DBT) in music therapy: a sequential explanatory study. J Music Ther. 2015. https:// doi.org/10.1093/jmt/thv002.

25. Cunningham $\mathrm{K}$, Wolbert $\mathrm{R}$, Lillie $\mathrm{B}$. It's about me solving my problems: clients' assessments of dialectical behavior therapy. Cogn Behav Pract. 2004 https://doi.org/10.1016/S1077-7229(04)80036-1.

26. Ditty MS, Landes SJ, Doyle A, Beidas RS. It takes a village: a mixed method analysis of inner setting variables and dialectical behavior therapy implementation. Admin Pol Ment Health. 2015. https://doi.org/10.1007/ s10488-014-0602-0.

27. Frederick JT, Comtois KA. Practice of dialectical behavior therapy after psychiatry residency. Acad Psychiatry. 2006. https://doi.org/10.1176/appi. ap.30.1.63.

28. Perseius K-I, Kåver A, Ekdahl S, Åsberg M, Samuelsson M. Stress and burnout in psychiatric professionals when starting to use dialectical behavioural therapy in the work with young self-harming women showing borderline personality symptoms. J Psychiatr Ment Health Nurs. 2007. https://doi.org/ 10.1111/j.1365-2850.2007.01146.x

29. Perseius K-I, Öjenhagen A, Ekdahl S, Åsberg M, Samuelsson M. Treatment of suicidal and deliberate self-harming patients with borderline personality disorder using dialectical behavioral therapy: the patients' and therapists' perceptions. Arch Psychiatr Nurs. 2003. https://doi.org/10.1053/S08839417(03)00093-1.

30. Sharma B, Dunlop B, Ninan PT, Bradley B. Use of dialectical behavior therapy in borderline personality disorder: a view from residency. Acad Psychiatry. 2007. https://doi.org/10.1176/appi.ap.31.3.218.

31. Swales MA, Taylor B, Hibbs RAB. Implementing dialectical behaviour therapy: programme survival in routine healthcare settings. J Ment Health. 2012. https://doi.org/10.3109/09638237.2012.689435.

32. Dimeff LA, Woodcock EA, Harned MS, Beadnell B. Can dialectical behavio therapy be learned in highly structured learning environments? Results from a randomized controlled dissemination trial. Behav Ther. 2011. https:// doi.org/10.1016/j.beth.2010.06.004.

33. Dimeff LA, Koerner K, Woodcock EA, Beadnell B, Brown MZ, Skutch JM, et al. Which training method words best? A randomized controlled trial comparing three methods of training clinicians in dialectical behavior therapy skills. Behav Res Ther. 2009. https://doi.org/10.1016/j.brat.2009. 07.011.

34. Hawkins KA, Sinha R. Can line clinicians master the conceptual complexities of dialectical behavior therapy? An evaluation of a state department of mental health training program. J Psychiatr Res. 1998. https://doi.org/10. 1016/S0022-3956(98)00030-2

35. Herschell AD, Lindheim OJ, Kogan JN, Celedonia KL, Stein BD. Evaluation of an implementation initiative for embedding dialectical behavior therapy in community settings. Evaluation Program Planning. 2014. https://doi.org/10. 1016/j.evalprogplan.2013.10.007.

36. Herschell AD, Kogan JN, Celedonia KL, Gavin JG, Stein BD. Understanding community mental health administrators' perspectives on dialectical behavior therapy implementation. Psychiatr Serv. 2009. https://doi.org/10. 1176/appi.ps.60.7989.

37. APA Gold Award. Using dialectical behavior therapy to help troubled adolescents return safely to their families and communities. Psychiatr Serv. 2004. https://doi.org/10.1176/appi.ap.55.10.1168 
38. Arroyo H, Steinberg E, Katz CL. "El grupo": bringing psychotherapy to an underserved population. Psychiatr Serv. 2012. https://doi.org/10.1176/appi. ps.20120p718

39. Baillie A, Slater S. Community dialectical behaviour therapy for emotionally dysregulated adults with intellectual disabilities. Adv Ment Health Intell Disabil. 2014. https://doi.org/10.1108/AMHID-05-2013-0033.

40. Barley WD, Buie SE, Peterson EW, Hollingsworth AS, Griva M, Hickerson SC, et al. Development of an inpatient cognitive-behavioral treatment program for borderline personality disorder. J Personal Disord. 1993. https://doi.org/ 10.1521/pedi.1993.7.3.232.

41. Award G. Integrating dialectical behavioral therapy into a community mental health program. Psychiatr Serv. 1998. https://doi.org/10.1176/ps.49.10.1338.

42. Engle E, Gadischkie S, Roy N, Nunziato D. Dialectical behavior therapy for a college population: applications at Sarah Lawrence college and beyond. J Coll Stud Psychother. 2013. https://doi.org/10.1080/87568225.2013.739014.

43. Kinsey K, Reed PG. Linking native American tribal policy to practice in mental health care. Nurs Sci Q. 2015. https://doi.org/10.1177/ 0894318414558616.

44. Lajoie T, Sonkiss J, Rich A. Development and clinical outcomes of a dialectical behavior therapy clinic. Acad Psychiatry. 2011. https://doi.org/10. 1176/appi.ap.35.5.325.

45. Lew M, Matta C, Tripp-Tebo C, Watts D. Dialectical behavior therapy (DBT) for individuals with intellectual disabilities: a program description. Ment Health Aspects Developmental Disabil. 2006;9:1-13.

46. Little L, Butler LS, Fowler J. Change from the ground up: bringing informeddialectical behavioral therapy to residential treatment. Resid Treat Child Youth. 2010. https://doi.org/10.1080/08865711003712527.

47. Morrissey C, Ingamells B. Adapted dialectical behaviour therapy for male offenders with learning disabilities in a high secure environment: six years on. J Learning Disabil Offending Behav. 2011. https:/doi.org/10.5042/jldob.2011.0024.

48. Sampl S, Wakai S, Trestman RL. Translating evidence-based practices from community to corrections: an example of implementing DBT-CM. J Behav Analysis Offender Victim Treat Prevention. 2010. https://doi.org/10.1037/h0100463.

49. Sunseri PA. Preliminary outcomes on the use of dialectical behavior therapy to reduce hospitalization among adolescents in residential care. Resid Treat ChildYouth. 2004. https://doi.org/10.1300/J007v21n04_06.

50. Vitacco MJ, Van Rybroek GJ. Treating insanity acquittees with personality disorders: implementing dialectical behavior therapy in a forensic hospital. J Forensic Psychol Pract. 2006. https://doi.org/10.1300/J158v06n02_01.

51. Wolpow S, Porter M, Hermanos E. Adapting a dialectical behavior therapy (DBT) group for use in a residential program. Psychiatr Rehabil J. 2000. https://doi.org/10.1037/h0095107.

52. Zinkler M, Gaglia A, Arokiadass SMR, Farhy E. Dialectical behaviour treatment: implementation and outcomes. Psychiatr Bull. 2007. https://doi. org/10.1192/pb.bp.106.011874.

53. Ben-Porath D, Peterson GA, Smee J. Treatment of individuals with borderline personality disorder using dialectical behavior therapy in a community mental health setting: clinical application and a preliminary investigation. Cogn Behav Pract. 2004. https://doi.org/10.1016/S10777229(04)80059-2.

54. Blennerhassett R, Bamford L, Whelan A, Jamieson S, O'Raghaillaigh JW. Dialectical behaviour therapy in an Irish community mental health setting. Ir J Psychol Med. 2009. https://doi.org/10.1017/S0790966700000227.

55. Bohus M, Haaf B, Stiglmayr C, Pohl U, Böhme R, Linehan M. Evaluation of inpatient dialectical-behavioral therapy for borderline personality disorder- a prospective study. Behav Res Ther. 2000. https://doi.org/10.1016/S00057967(99)-00103-5.

56. Brassington J, Krawitz R. Australasian dialectical behaviour therapy pilot outcome study: effectiveness, utility and feasibility. Australas Psychiatry. 2006. https://doi.org/10.1080/j.1440-1665.2006.02285.x.

57. Comtois KA, Elwood L, Holdcraft LC, Smith WR, Simpson TL. Effectiveness of dialectical behavior therapy in a community mental health center. Cogn Behav Pract. 2007. https://doi.org/10.1016/j.cbpra.2006.04.023

58. Eccleston L, Sorbello L. The RUSH program -- real understanding of selfhelp: a suicide and self-harm prevention initiative within a prison setting. Aust Psychol. 2002. https://doi.org/10.1080/00050060210001706926.

59. Hjalmarsson E, Kåver A, Perseius K-I, Cederberg K, Ghaderi A. Dialectical behaviour therapy for borderline personality disorder among adolescents and young adults: pilot study, extending the research findings in new settings and cultures. Clin Psychol. 2008. https://doi.org/10.1080/ 13284200802069035
60. James S, Freeman KR, Mayo D, Riggs ML, Morgan JP, Schaepper MA, et al. Does insurance matter? Implementing dialectical behavior therapy with two groups of youth engaged in deliberate self-harm. Admin Pol Ment Health. 2015. https://doi.org/10.1007/s100488-014-0588-7.

61. Katz LY, Cox BJ, Gunasekara S, Miller AL. Feasibility of dialectical behavior therapy for suicidal adolescent inpatients. J Am Acad Child Adolesc Psychiatry. 2004. https://doi.org/10.1097/00004583-200403000-00008.

62. Kerr PL, Muehlenkamp JJ, Larsen MA. Implementation of DBT-informed therapy at a rural university training clinic: a case study. Cogn Behav Pract. 2009. https://doi.org/10.1016/j.cbpra.2008.09.003.

63. Nee C, Farman S. Female prisoners with borderline personality disorder: some promising treatment developments. Crim Behav Ment Health. 2005 https://doi.org/10.1002/cbm.33.

64. Nelson-Gray RO, Keane SP, Hurst MR, Mitchell JT, Warburton JB, Chok JT, et al. A modified DBT skills training program for oppositional defiant adolescents: promising preliminary findings. Behav Res Ther. 2006. https:// doi.org/10.1016/j.brat.2006.01.004.

65. Koons CR, Chapman AL, Betts BB, O'Rourke B, Morse N, Robins CJ. Dialectical behavior therapy adapted for the vocational rehabilitation of significantly disabled mentally ill adults. Cogn Behav Pract. 2006. https://doi.org/10.1016/ j.cbpra.2005.04.003.

66. Pasieczny N, Connor J. The effectiveness of dialectical behaviour therapy in routine public mental health settings: an Australian controlled trial. Behav Res Ther. 2011. https://doi.org/10.1016/j.brat.2010.09.006.

67. Prendergast N, McCausland J. Dialectic behaviour therapy: a 12-month collaborative program in a local community setting. Behav Change. 2007. https://doi.org/10.1375/bech.24.1.25.

68. Rathus JH, Miller AL. Dialectical behavior therapy adapted for suicidal adolescents. Suicide Life Threat Behav. 2002. https://doi.org/10.1521/suli.32. 2.146.24399.

69. Trupin EW, Stewart DG, Beach B, Boesky L. Effectiveness of a dialectical behaviour therapy program for incarcerated female juvenile offenders. Child Adolesc Ment Health. 2002. https://doi.org/10.1111/1475-3588.00022.

70. van den Bosch LMC, Verheul R, Schippers GM, van den Brink W. Dialectical behavior therapy of borderline patients with and without substance use problems. Implementation and long-term effects. Addict Behav. 2002. https://doi.org/10.1016/S0306-4603(02)00293-9.

71. Woodberry KA, Popenoe EJ. Implementing dialectical behavior therapy with adolescents and their families in a community outpatient clinic. Cogn Behav Pract. 2008. https://doi.org/10.1016/j.cbpra.2007.08.004.

\section{Ready to submit your research? Choose BMC and benefit from:}

- fast, convenient online submission

- thorough peer review by experienced researchers in your field

- rapid publication on acceptance

- support for research data, including large and complex data types

- gold Open Access which fosters wider collaboration and increased citations

- maximum visibility for your research: over $100 \mathrm{M}$ website views per year

At $\mathrm{BMC}$, research is always in progress.

Learn more biomedcentral.com/submission 